\title{
Modular Multilevel Converter Based STATCOM Topology Suitable for Medium-Voltage Unbalanced Systems
}

\author{
Hassan Mohammadi Pirouz ${ }^{\dagger}$ and Mohammad Tavakoli Bina* \\ ${ }^{\dagger *}$ School of Electrical and Computer Eng., K. N. Toosi University of Technology, Tehran, Iran
}

\begin{abstract}
This paper discusses a transformerless shunt static compensator (STATCOM) based on a modular multilevel converter (MMC). It introduces a new time-discrete appropriate current control algorithm and a phase-shifted carrier modulation strategy for fast compensation of the reactive power and harmonics, and also for the balancing of the three-phase source side currents. Analytical formulas are derived to demonstrate the accurate mechanism of the stored energy balancing inside the MMC. Various simulated waveforms verify that the MMC based STATCOM is capable of reactive power compensation, harmonic cancellation, and simultaneous load balancing, while controlling and balancing all of the DC mean voltages even during the transient states.
\end{abstract}

Key Words: DC voltage balancing, Medium voltage, Modular multilevel converter, STATCOM, Unbalanced currents

\section{INTRODUCTION}

Nowadays, multilevel converters are used like the power stages in STATCOMs [1], [2], due to their advantages over other converter topologies. The voltage stresses can be reduced when the number of levels increases, the power switches are driven with a low commutation frequency and multilevel converters can synthesize a voltage waveform with a very low harmonic content [3]-[8]. Compared with diode clamped multilevel converters or flying capacitor multilevel converters, the cascaded multilevel converters can be directly connected to a medium-voltage network without a bulky step up transformer, resulting in cost and weight reductions [6]-[9]. However, they have some restrictions [7]-[11] when the fast compensation of large, fluctuating unbalanced loads, such as electric traction systems [12], is required [9].

Modular multilevel converters [13] (MMC) have recently been proposed as an alternative to conventional multilevel converters in medium voltage applications [14], [15]. They provide a viable approach to constructing a reliable and cost effective STATCOM [11], with an increased number of levels capable of eliminating the coupling transformer and replacing it with cheap reactors to allow a power exchange with the power system [15]. In addition, it can operate continuously under unbalanced conditions, it is capable of surviving symmetrical and asymmetrical faults without increasing the risk of system collapse and it has fault management capability.

The focus of this paper is to realize a transformerless

Manuscript received Mar. 4, 2010; revised Jun. 19, 2010

†Corresponding Author: pirouz@ee.kntu.ac.ir

Tel:+98-21-88462174, Fax: +98-21-88462066, K. N. Toosi Univ.

* School of Electrical and Computer Eng., K. N. Toosi Univ., Iran
STATCOM, based on a MMC for the compensation of a nonlinear unbalanced load in a medium-voltage level. For this purpose, a control strategy based on the instantaneous power theory is developed for extracting the compensating current signals. Then, a new real-time current control technique is introduced for the MMC, based on the predictive control method. An appropriate switching modulation technique is applied to the MMC, keeping the stored energy in all of the legs balanced, even if the converter currents are unbalanced and the network voltages are slightly distorted. Analytical formulas are derived to demonstrate the accurate mechanism of the DC-link voltage balancing. Simulations are conducted to prove the effectiveness of the proposed controller and the topology of the MMC based STATCOM.

\section{Power Circuit Description}

\section{A. Main circuit structure}

The basic circuit structure of a four-wire STATCOM based on a MMC is depicted in Fig. 1. Unlike conventional multilevel converters such as diode clamped or flying capacitor multilevel converters, there is no common DC-link capacitor in the configuration of the proposed STATCOM topology. The MMC is comprised of two polarized star-connected half-bridge cascaded converters (HBCC), which are connected to the network in parallel. While one HBCC has a negative common link (NLHBCC), the other has a positive common link (PL-HBCC), and the negative and positive links are floating points. Each leg of both of the HBCCs consists of a number of series-connected half-bridge modules (HBM), and the legs are connected in a star structure. Thus, each HBCC can be directly connected 


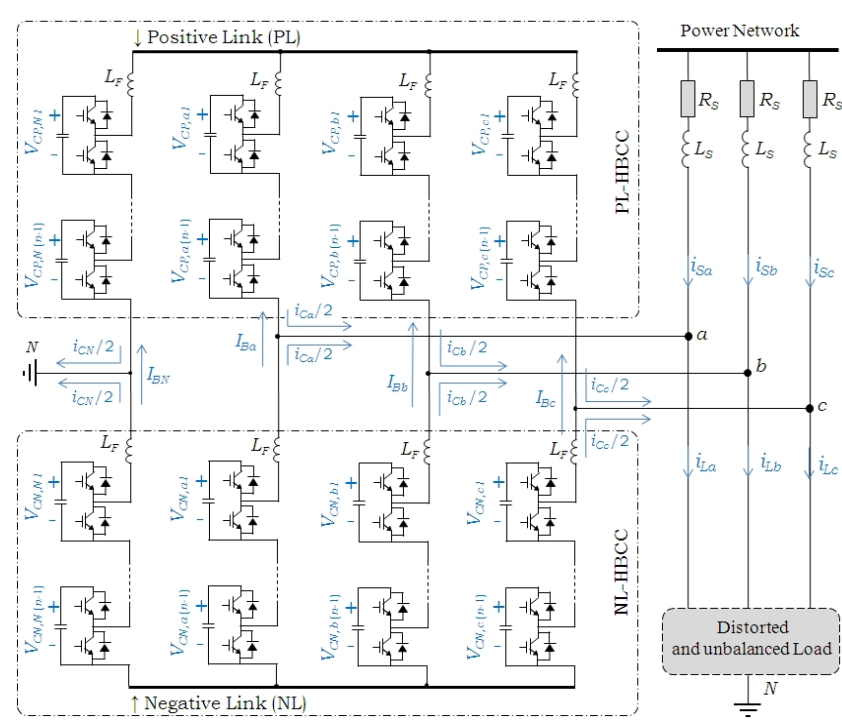

Fig. 1. Circuit structure of the MMC based STATCOM and the way of its connection to the network.

to a medium-voltage network without a coupling transformer. In four-wire load compensation, both of the star-connected HBCCs have four similar legs. To compensate a three-wire unbalanced load, the converter can also be composed of two three-leg polarized HBCCs. Both the NL-HBCC and the PLHBCC have the same power rating as well as the same current contribution to the STATCOM. In fact, each HBCC can be independently applied to the network as a STATCOM, when the required compensating currents are balanced. Under unbalanced conditions, the flow of the negative-sequence currents on the output side of the MMC causes circulating current flow among the HBCC legs of the converter. The most important effect of the circulating current is the energy transfer between the legs. Therefore, it is imperative that both the PL-HBCC and the NL-HBCC in the shape of one MMC are applied to the network as a STATCOM, when the required compensating currents are unbalanced. In this condition, the stored energy of all of the legs of the MMC can be balanced, by applying an appropriate modulation scheme.

\section{B. Half-bridge cascaded converters}

An $n$-level HBCC is defined by the available $(n-1)$ identical HBMs cascaded in each leg of that HBCC. All of the $n$-level legs are connected to the network using an inductive filter $\left(L_{F}\right)$. In addition, all of the HBMs have the same semiconductor ratings as well as identical DC-link capacitances. Therefore, each HBM can be assumed to be an identical two-terminal device. Voltage regulation of the DClink capacitors is achieved without any additional connections or energy transfer circuits to the associated HBM. Each HBM is capable of producing either $V_{C m}$ (the DC-link capacitor voltage of the module) or 0 volt at any given instance. Thus, the resultant voltage of a $(n-1)$ cascaded HBM varies between $\left[0, V_{D C M}\right]$, where $V_{D C M}=(n-1) V_{C m}$.

The voltage across a cascaded HBM, in all of the legs of each HBCC, includes a DC component and an AC component, as shown in Fig. 2. The value of the $\mathrm{AC}$ voltage

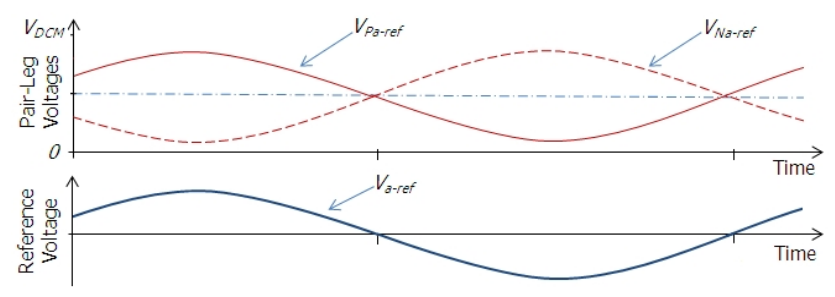

Fig. 2. Reference voltages of the NL-HBCC leg and the PL-HBCC leg against the pair-leg reference voltage, all for phase $a$.

component must be the same for the corresponding legs of the NL-HBCC and the PL-HBCC, while the value of the DC voltage component must be in the inverted form for them. As a result, irrespective of the voltages on the filter inductors, the average voltage between the positive-link and the negative-link $\left(V_{P L}-V_{N L}\right)$ is always $V_{D C M}$. Thus, the average voltage between the positive-link and the neutral point $(N)$ is $V_{P L}=+V_{D C M} / 2$, while the average voltage between negative-link and $N$ is $V_{N L}=-V_{D C M} / 2$. Although the voltage on a leg has a DC component, there exists no DC component on the line-to-line voltage or the line-to-neutral voltage in both HBCCs. The instantaneous voltage on any two terminals of an $\mathrm{HBCC}$ is dictated by the difference between the cascaded HBM voltages of each of the legs connected to those terminals. Therefore, regardless of the filter inductor voltages, the line-to-line voltages of an HBCC can be adjusted within $\left[-V_{D C M},+V_{D C M}\right]$. To enable compensation of the inductive loads, a value of $V_{D C M}$ must be chosen that is greater than the peak-to-peak amplitude of the line voltage [16]. In addition, both the NL-HBCC leg and the PL-HBCC leg connected to the same phase are controlled so that they constantly supply half of the MMC current per phase.

\section{Balancing currents}

Under unbalanced conditions, the flow of the negativesequence currents on the output side of the MMC causes the DC currents to flow along the HBCC legs of the converter. In this condition, the energy stored in the HBM capacitors of the leg supplying active power wants to be reduced, while it wants to be increased in the leg consuming active power. The energy stored in both of the legs connected to one phase (pair-leg) is equal, because each leg provides half of the output current in the corresponding phase. Nonetheless, the energy stored in the four pair-legs of the MMC may have different values, in the unbalanced condition. As a result, the direct balancing currents $\left(I_{B}\right)$, flow from the over-charged pair-legs towards the undercharged pair-legs. Under such circumstances, the leg currents of the pair-leg connected to phase $x$ are equal to:

$$
\left[\begin{array}{c}
i_{N x} \\
i_{P x}
\end{array}\right]=\left[\begin{array}{c}
\frac{i_{C x}}{2}+I_{B x} \\
\frac{i_{C x}}{2}-I_{B x}
\end{array}\right](x=a, b \text { or } c)
$$

where, $i_{N x}, i_{P x}, i_{C x}$ and $I_{B x}$ are the NL-HBCC leg current, the PL-HBCC leg current, the STATCOM output current and the pair-leg balancing current, respectively, for phase $x$. In addition, in a four-leg MMC, the current of the NL-HBCC 

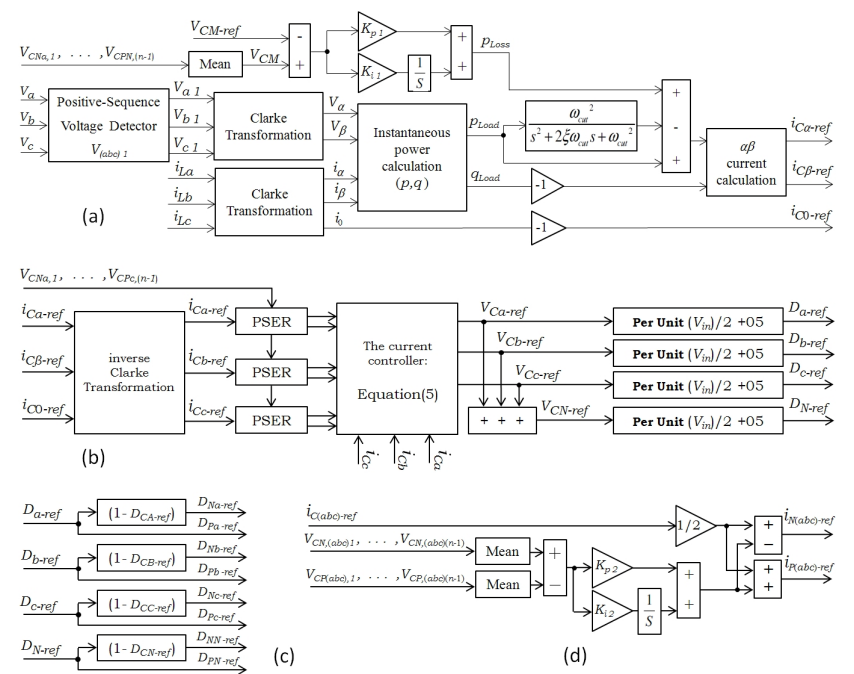

Fig. 3. Proposed controller for the MMC based STATCOM: (a) derivation of the three-phase reference currents in $\alpha \beta 0$ coordinates, (b) the current controller and reference duty cycle extraction, (c) duty cycle extraction to generate switching modulation signals for each HBM, (d) the Pair-leg Stored Energy Regulator (PSER) diagram.

leg and the PL-HBCC leg connected to the neutral can be obtained as follows:

$$
\left[\begin{array}{c}
i_{N N} \\
i_{P N}
\end{array}\right]=-\left[\begin{array}{c}
i_{N a}+i_{N b}+i_{N c} \\
i_{P a}+i_{P b}+i_{P c}
\end{array}\right] .
$$

The balancing current magnitude in a pair-leg, $I_{B x}$ depends on the value of the active power interchanged between the network and that pair-leg. Applying Kirchhoff's current law (KCL) for each of the HBCCs leads to:

$$
I_{B a}+I_{B b}+I_{B c}+I_{B N}=0 .
$$

This means that the sum of the total converter balancing currents will always be zero. In the presence of an appropriate modulation technique, the balancing current makes the stored energy at all of the legs remain balanced. In the steady state condition, the value of the balancing current through a pair-leg does not have an effect on the output current of the STATCOM in the corresponding phase. Meanwhile, any balancing current fluctuations are attenuated through the inductance $L_{F}$. Whenever an abrupt change occurs in the converter currents, the filter inductors damp the sudden rise in the balancing current $I_{B x}$.

The filter inductors, in all legs are identical and the inductance $L_{F}$ is calculated according to the maximum permitted ripple on the output currents. The value of the required inductance can be calculated through the following equation:

$$
L_{F}=\frac{V_{D C M}}{(n-1) f_{C} \Delta i_{C, \max }}
$$

where $\Delta i_{C, \max }$ is the maximum allowable ripple on the output current and $f_{C}$ is the switching frequency of each HBM.

\section{Control Method}

The main challenges associated with MMC based STATCOM control are shaping the output phase currents, balancing the DC-link voltages of all of the HBMs, and keeping the DClink voltages at the desired reference value. However, this can become difficult if the converter currents have zero or negative sequence components, or the series HBMs have slightly different characteristics. When implementing a medium voltage MMC, even the parasitic stray capacitances to the Earth can lead to unwanted scaling effects and unequal voltage distribution among the series connected HBMs. A basic diagram of the proposed controller is shown in Fig. 3. The diagram consists of both a reference current extractor and a predictive current controller.

\section{A. Reference signals calculation}

The general instantaneous power theory [17] introduces the reference currents for each phase of the MMC based STATCOM as described in Fig. 3(a). The objective of this compensation theory is to make the source currents completely sinusoidal and balanced, i.e. in phase with the fundamental positive sequence component of the source voltage. The reference current can be obtained for both unbalanced load conditions and unbalanced voltages conditions, simultaneously. By measuring the three-phase voltages of the point of common coupling (PCC), the reference voltages of the legs in each pairleg can be calculated. It can be presented in a discrete form by a good approximation within the switching time period $[k$, $k+1$ ], as below:

$$
\begin{aligned}
& {\left[\begin{array}{l}
V_{N x, r e f}(k) \\
V_{P x, \text { ref }}(k)
\end{array}\right]=} \\
& \quad\left[\begin{array}{c}
V_{x}(k-1)-\frac{L_{F}\left(i_{C x, r e f}(k)-i_{C x}(k-1)\right) f_{s}}{L_{F}\left(i_{C x, r e f}(k)-i_{C x}(k-1)\right) f_{s}} \\
2
\end{array}\right] \\
& \quad+\left[\begin{array}{c}
\frac{V_{D C M}}{2} \\
-\frac{V_{D C M}^{2}}{2}
\end{array}\right]
\end{aligned}
$$

where, $V_{N x, r e f}$ and $V_{P x, r e f}$ are the predicted reference voltages for the NL-HBCC leg and the PL-HBCC leg, respectively; $V_{x}$ is the point of common coupling voltage; $i_{C x, \text { ref }}$ denotes the reference current of the STATCOM; and $f_{S}$ is the sampling frequency of the control unit; for the legs connected to phase $x$. The balancing current, $I_{B x}$ is the same in both legs of a pair-leg for steady state operation of the STATCOM, ignoring the derivation of $I_{B x}$ in (5). Neglecting the resistance associated with $L_{F}$, (5) can be employed as a predictive current controller for the STATCOM to track the reference currents. In a four-leg MMC, the predicted reference voltages for the NL-HBCC leg and the PL-HBCC leg connected to the neutral point $N$ are determinate as follows:

$$
\left[\begin{array}{l}
V_{N N, \text { ref }}(k) \\
V_{P N, \text { ref }}(k)
\end{array}\right]=\left[\begin{array}{l}
V_{N a, r e f}(k)+V_{N b, r e f}(k)+V_{N c, r e f}(k) \\
V_{P a, r e f}(k)+V_{P b, r e f}(k)+V_{P c, r e f}(k)
\end{array}\right]
$$

Therefore, the duty cycle for all of the HBMs in each leg can be obtained from (5) and (6), by real-time measuring of the currents and voltages of the converter and load, as shown in Fig. 3(b). 


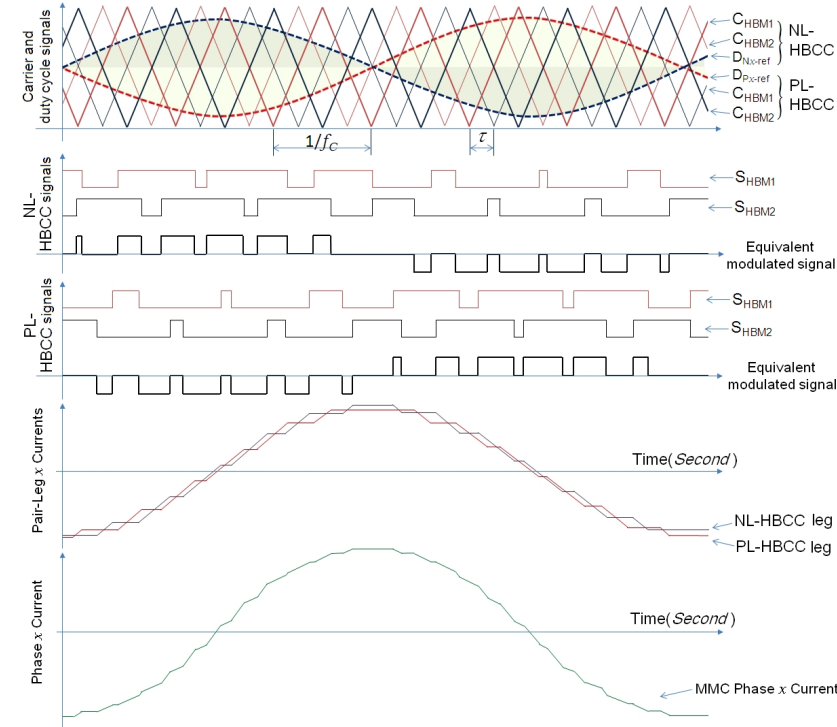

Fig. 4. Illustration of applied phase-shifted PWM modulation technique to create switching signals for all HBM in a pair-leg, assuming each leg of the MMC includes two HBM.

\section{B. HBM switching modulation signals}

Among the various pulse programming methods, the carrier based pulse width modulation (PWM) methods are the preferred approaches in multilevel converters due to their fixed switching frequencies and their implementation simplicity. Phase-shifted PWM (PS-PWM) is the most commonly used modulation technique for cascaded multilevel converters, because it offers an even power distribution among all of the HBMs, and it is very easy to implement independent of the number of series HBMs [18]. This modulation shifts the phase of each carrier signal in a proper angle to reduce the harmonic content of the passing current from each leg and to lower the output current ripple of the MMC (see Fig. 4). Although the carrier signals used for each HBM have a similar shape, they are relatively shifted to each other as follows:

$$
\tau=\frac{1}{2(n-1) f_{C}}
$$

where $\tau$ is the time interval between two adjacent carrier signals and $f_{C}$ is the frequency of each carrier signal (actually the switching frequency of each HBM). While each HBM in a leg has an independent carrier signal, the reference signal is shared by all of the HBMs in a series. The switching pattern for each HBM is obtained by comparing the reference duty cycle signal with the carrier signal related to that HBM. To reduce the output current ripple of the MMC, the carrier signals for the NL-HBCC legs are shifted by $180^{\circ}$ in comparison with those of the PL-HBCC legs, as shown in Fig. 4. Therefore, the output current ripple of the MMC can reciprocally be canceled up to $50 \%$ in comparison with the ripples of each HBCC.

\section{DC-Link Voltage BALANCING}

\section{A. Self energy balancing inside the MMC}

The instantaneous power of both legs in a pair-leg connected to phase $x$ of the network, can be calculated using (1) and (5). Over the network frequency (i.e. $50 \mathrm{~Hz}$ or $60 \mathrm{~Hz}$ ), the instantaneous power of each leg has a DC component along with an alternative component as follows:

$$
\left[\begin{array}{l}
P_{N x} \\
P_{P x}
\end{array}\right]=\left[\begin{array}{c}
\bar{P}_{N x} \\
\bar{P}_{P x}
\end{array}\right]+\left[\begin{array}{c}
\tilde{P}_{N x} \\
\tilde{P}_{P x}
\end{array}\right]
$$

Each part of (8) is equal to:

$$
\begin{aligned}
& {\left[\begin{array}{l}
\bar{P}_{N x} \\
\bar{P}_{P x}
\end{array}\right]=} \\
& {\left[\begin{array}{c}
\frac{i_{C x} \cdot V_{x}}{2}-\frac{i_{C x} \cdot L_{F}\left(i_{C x, r e f}-i_{C x}\right) f_{s}}{4}+\frac{I_{B x} V_{D C M}}{2} \\
\frac{i_{C x} \cdot V_{x}}{2}-\frac{i_{C x} \cdot L_{F}\left(i_{C x, r e f}-i_{C x}\right) f_{s}}{4}+\frac{I_{B x} V_{D C M}}{2}
\end{array}\right]} \\
& {\left[\begin{array}{c}
\tilde{P}_{N x} \\
\tilde{P}_{P x}
\end{array}\right]=} \\
& {\left[\begin{array}{c}
i_{B x} \cdot V_{x}-\frac{i_{B x} \cdot L_{F}\left(i_{C x, r e f}-i_{C x}\right) f_{s}}{2}+\frac{I_{C x} V_{D C M}}{4} \\
-i_{B x} \cdot V_{x}+\frac{i_{B x} \cdot L_{F}\left(i_{C x, r e f}-i_{C x}\right) f_{s}}{2}-\frac{I_{C x} V_{D C M}}{4}
\end{array}\right] .}
\end{aligned}
$$

Considering (9), both of the components in the DC component of (8) are similar. Therefore, the average active power is the same for both legs of a pair-leg, over the network frequency. Moreover, regarding (2), the neutral current of the converter is equally divided between the two legs connected to the neutral, in a four-leg MMC. Accordingly, the instantaneous power for the NL-HBCC leg and the PL-HBCC leg connected to the neutral can be determinate as follows:

$$
\begin{aligned}
& {\left[\begin{array}{l}
P_{N N} \\
P_{P N}
\end{array}\right]=\left[\begin{array}{l}
V_{P L} \\
V_{N L}
\end{array}\right]\left[\begin{array}{ll}
\frac{i_{C N}}{2}-I_{B N} & \frac{i_{C N}}{2}+I_{B N}
\end{array}\right]=} \\
& {\left[\begin{array}{c}
\frac{V_{D C M}}{2} \frac{i_{C N}}{2} \\
-\frac{V_{D C M}}{2} \frac{i_{C N}}{2}
\end{array}\right]-\left[\begin{array}{c}
\frac{V_{D C M}}{2} I_{B N} \\
\frac{V_{D C M}}{2} I_{B N}
\end{array}\right]}
\end{aligned}
$$

Considering (11), the DC component of the instantaneous power is equal to $\left(V_{D C M} I_{B N}\right) / 2$, which is the same for both legs connected to the neutral. As a result, the stored energy in both legs of each pair-leg is equal. On the other hand, the stored energy in all of the pair-legs becomes equal, due to the presence of the balancing currents, resulting in an energy balance among all of the pair-legs in the converter. In fact, all of the pair-legs are connected together in parallel. Therefore, the MMC theoretically has the ability of self energy balancing among all of the legs, even in unbalanced conditions. To regulate the total stored energy inside the converter, so that it is equal to a predetermined reference value, a DC voltage regulator unit is added to the reference output power of the STATCOM as shown in Fig. 3(a). As a result, the mean value of all of the DC-link capacitor voltages is regulated toward a certain value by estimating the power losses through the DC voltage regulator unit.

However, the energy stored in both legs connected to the same phase may be a little different, due to the non-ideal nature of the converter elements. This may be eliminated by using an appropriate local controller to adjust the current contribution of both legs connected to the same phase, as shown in Fig. 3(d). There are four pair-leg stored energy regulators (PSER) in the MMC, that regulate the stored energy of both legs in a pair-leg. 


\section{B. Capacitor voltage balancing in the cascaded HBM}

Each HBM has two complementary switches in series, providing either the DC-link voltage or zero for the AC output. When the AC output voltage of a HBM in the NL-HBCC is set to its DC-link voltage, the capacitor voltage of the HBM, decreases for a positive input current $\left(i_{N x}>0\right)$, and increases for a negative input current $\left(i_{N x}<0\right)$. Conversely, in the PL-HBCC, the capacitor voltage increases for a positive input current $\left(i_{P x}>0\right)$ and decreases for a negative input current $\left(i_{P x}<0\right)$. When the output voltage of a HBM is set to zero, the capacitor voltage will not change in both of the HBCCs. These switching effects can be used for capacitor voltage balancing among all of the series HBMs in a leg. For this purpose, the measured capacitor voltages of the legs are sorted in ascending order during each of the switching periods. The sign of the leg current and the number of the HBM that are permanently set to its DC-link voltage determine which HBM should be selected [19].

\section{Total energy stored inside the MMC}

The capacitance of the DC-link capacitors in each HBM is determined on the basis of the maximum permitted variations of the DC-link voltage. The minimum DC capacitance requirement for a HBM can be calculated by using the following equation:

$$
C_{m}=\frac{i_{H B M}}{f_{C} \cdot \Delta V_{C m, \max }}
$$

where $\Delta V_{C m, \max }$ is the maximum allowable voltage ripple on the DC-link among all of the HBMs., $i_{H B M}$, is the nominal current rating for each HBM and specified by:

$$
i_{H B M}=\left.\frac{\max \left(i_{x}\right)}{2}\right|_{x=a, b, c, N}
$$

where $\max \left(i_{x}\right)$ is the maximum output current coming out of the converter terminals. The total energy stored in all of the distributed capacitors of the MMC can be calculated using (12) and (13) as bellow:

$$
\begin{aligned}
& E_{t}=8(n-1)\left[\frac{1}{2} C_{m} V_{C m}^{2}\right]= \\
& \frac{4 i_{H B M} V_{D C M}^{2}}{(n-1) f_{C} \Delta V_{C m, \max }}=\left.\frac{2 \max \left(i_{x}\right) V_{D C M}^{2}}{f_{C} \Delta V_{D C M}}\right|_{x=a, b, c, N} .
\end{aligned}
$$

It is known that the total energy stored in a conventional multilevel STATCOM with a common DC-link such as a diode clamped multilevel STATCOM is equal to (15):

$$
E_{t}=\left[\frac{1}{2} C_{D C} V_{D C M}^{2}\right]=\frac{\max \left(i_{D C}\right) V_{D C M}^{2}}{2 f_{C} \Delta V_{D C M}}
$$

where $C_{D C}$ is the total capacitance of the common DClink and $i_{D C}$ is the instantaneous DC-link current. The value of $i_{D C}$ depends on the negative sequence currents of the converter [20], and taking the worst case leads us to have $i_{D C}$ $=\max \left(i_{x}\right)$. Therefore in the same converter rating and voltage ripple condition, the total energy stored in the distributed capacitors of the MMC based STATCOM is four times higher than the energy stored in a conventional multilevel STATCOM having one central capacitor in the common DC-link.
TABLE I

Circuit Parameters Used for Simulation

\begin{tabular}{|l|c|c|}
\hline Converter power capacity & $S$ & $10 \mathrm{MVA}$ \\
RMS line-to-line voltage at PCC & $V_{L L}$ & $25 \mathrm{kV}$ \\
Source inductance & $L_{S}$ & $12.5 \mathrm{mH}(\% 6.25)$ \\
Source resistance & $R_{S}$ & $0.7 \Omega(\% 1.12)$ \\
Number of HBM in each leg & $n$ & 25 \\
Inductance of filter inductor & $L_{F}$ & $3 \mathrm{mH}(\% 1.5)$ \\
DC-Link voltage of each HBM & $V_{C m}$ & $3 \mathrm{kV}$ \\
DC-Link capacitors specification & $C / V$ & $1.1 \mathrm{mF} / 3.3 \mathrm{kV}$ \\
Carrier frequency & $f_{C}$ & $1000 \mathrm{~Hz}$ \\
Equivalent switching frequency & $50 f_{C}$ & $50 \mathrm{kHz}$ \\
RMS current rating of the HBM & $i_{H B M}$ & $300 \mathrm{~A}$ \\
\hline \multicolumn{2}{|c|}{ on a three-phase $25 \mathrm{kV}, 10 \mathrm{MVA}, 50 \mathrm{~Hz}$ base }
\end{tabular}

TABLE II

Control Parameters USEd for Simulation

\begin{tabular}{|l|c|c|}
\hline Proportional gain of DC voltage regulator & $K_{p 1}$ & $10^{5}$ \\
Integral gain of DC voltage regulator & $K_{i 1}$ & $10^{3}$ \\
Proportional gain of the PSER & $K_{p 2}$ & $10^{-4}$ \\
Integral gain of the PSER & $K_{i 2}$ & $10^{-2}$ \\
Damping ratio of low pass filter & $\xi$ & 0.707 \\
Cut off frequency of low pass filter & $\omega_{c u t}$ & $10 \pi \mathrm{rad} / \mathrm{s}$ \\
\hline
\end{tabular}

\section{Simulation Study}

The proposed control algorithm is implemented in MATLAB, and the power circuit is simulated using PSIM to preliminarily verify the effectiveness of the proposed MMC based STATCOM. Assuming an electrical railway application, a $25 \mathrm{kV}$ network having a distorted unbalanced load is considered for compensation. A single branch load having $9.41 \%$ THD is connected between two phases of the PCC. Each leg of the compensator has twenty-five HMBs. All of the HBMs have a DC storage capacitor with a nominal DC voltage of $3.3 \mathrm{kV}$. However, the DC-link voltage of each HBM is set $3 \mathrm{kV}$. Therefore, the voltage between the negative and positive links will be $V_{D C M}=75 \mathrm{kV}$. The PS-PWM modulation technique, with a carrier frequency of $f_{C}=1000 \mathrm{~Hz}$, is applied to all of the HBMs. Hence, considering Fig. 4, the equivalent switching frequency is $50 \mathrm{kHz}$ from the MMC output current point of view, while each switch of the HBMs in the MMC based STATCAM is working at $1000 \mathrm{~Hz}$. The carrier frequency is selected based upon several factors such as the switch type, the level of output voltage and the allowable THD content of the output current. Table 1 and 2 summarize the circuit parameters and the control parameters that are used in the simulation study.

The load current waveforms and the source-end current waveforms, before and after compensation, are shown in Fig. 5 . Before the start time $\left(t_{S}=0.1 \mathrm{~S}\right)$, the compensator is deactivated, and then after $t_{S}$, the source-end currents are balanced and the harmonics are almost eliminated by the proposed compensator. In addition, the source-end currents become in phase with the fundamental of the positive sequence voltage and they don't contain reactive power components. Also the voltages at the PCC become harmonic free and balanced after 

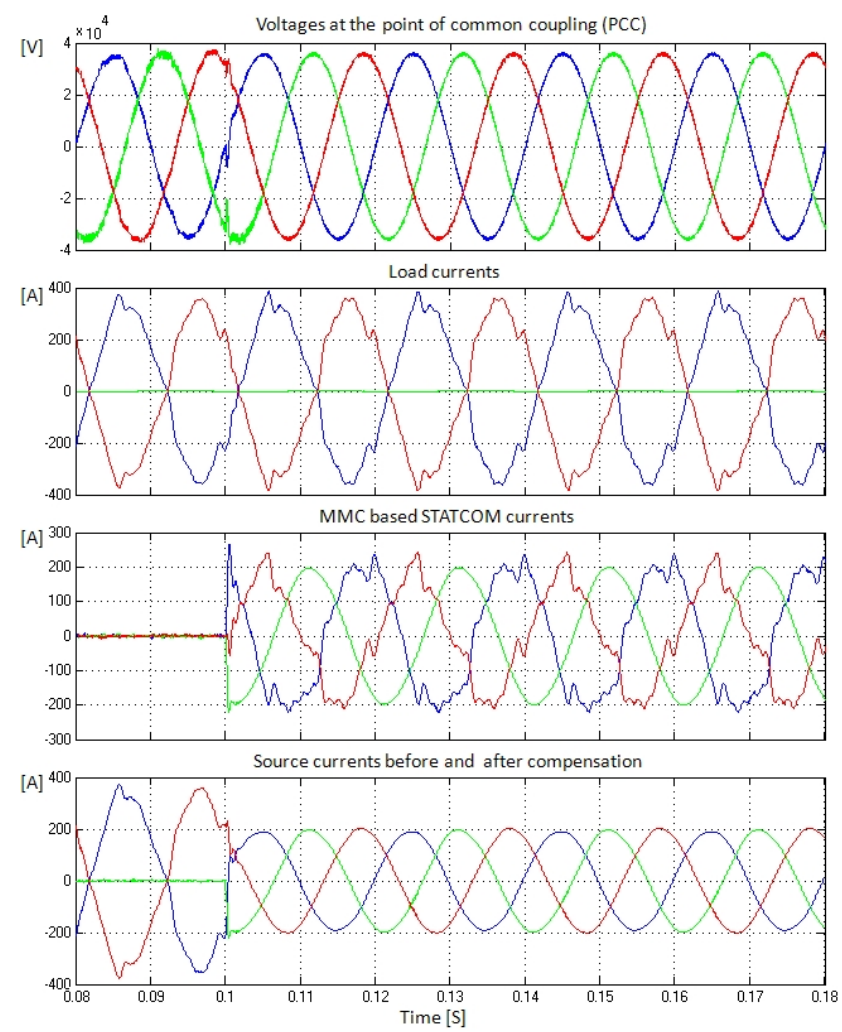

Fig. 5. Voltage waveforms at the PCC, and current waveforms in the load side and source side, befor and after compensation by the MMC based STATCOM.
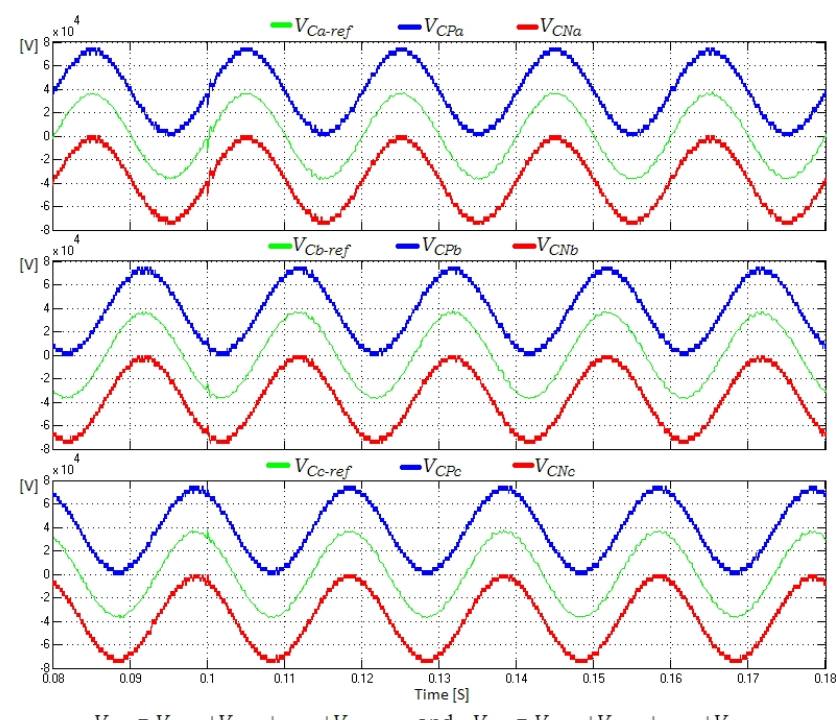

$V_{C P x}=V_{C P, x 1}+V_{C P, x 2}+\ldots+V_{C P, x(n-1)}$ and $V_{C N X}=V_{C N, x 1}+V_{C N, x 2}+\ldots+V_{C N, x(n-1)}$

Fig. 6. Voltage of the PL-HBCC and the NL-HBCC legs, neglecting the series inductor voltage.

$t_{S}$. Fig. 6 shows the voltage waveforms of the PL-HBCC and the NL-HBCC legs, neglecting the filter inductor voltages in the MMC. Fig. 7 shows the DC-link voltages of the HBMs in both the NL-HBCC and the PL-HBCC before and after $t_{S}$. All of the HBMs in a leg have the same variation because of the capacitor voltage balancing algorithm in each leg. Because of the unbalanced output current of the MMC, there is a slight difference between the average values of the DC-link voltages of the HBMs in different pair-legs. The pair-leg connected
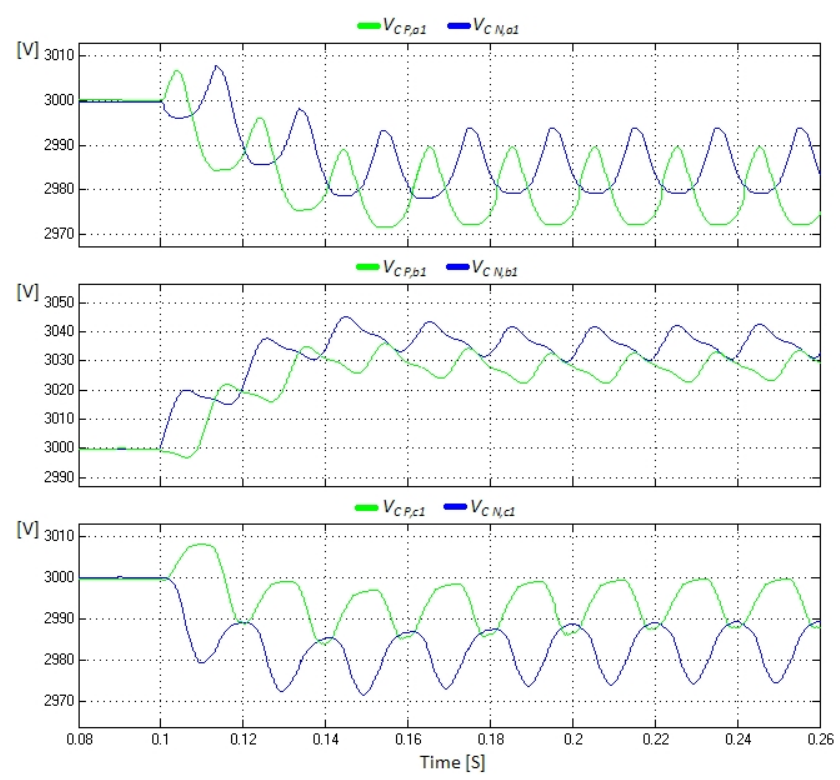

Fig. 7. HBM DC-link voltage ripples, before and after compensation of the unabalaned and distorted load.
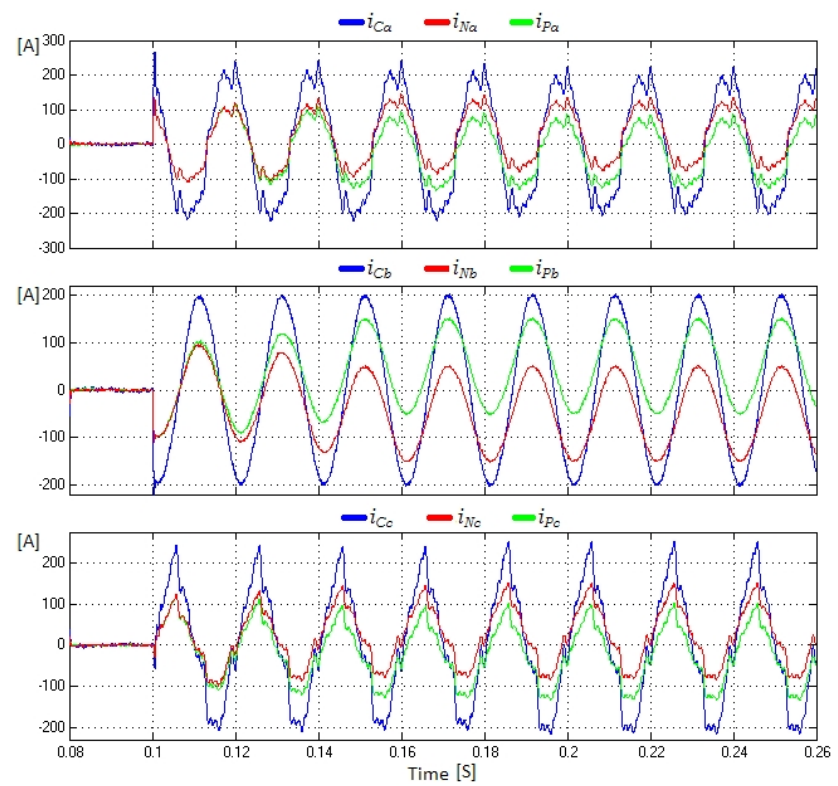

Fig. 8. The MMC legs currents before and after compensation of the unbalanced and distorted load, and their transient behavior.

to phase $b$ consumes the active power, while the pair-legs connected to phase $a$ and phase $c$, produce the active power balancing of the source currents. As a result, the average values of $V_{C P, b 1}$ and $V_{C N, b 1}$ will be increased and the average values of $V_{C P, a 1}, V_{C N, a 1}, V_{C P, c 1}$, and $V_{C N, c 1}$ will be reduced. As see in Fig. 8, the balancing currents flow from the pair-leg of phase $b$ to the pair-legs of phase $a$ and phase $c$, because of the DC voltage differences. Considering Fig. 8, the magnitude of the balancing current in phase $b$ is measured at about $I_{B b}$ $=50 \mathrm{~A}$, while in phase $a$ and phase $c$ it is measured at about $I_{B a}=I_{B c}=-25 \mathrm{~A}$. As a result, the proposed controller can regulate all of the DC-link mean voltages at a predetermined level while the output currents of the MMC based STATCOM are unbalanced and distorted. 


\section{CONCLUSiOnS}

This paper presents a novel high-power STATCOM based on a modular multilevel converter to compensate an unbalanced and distorted medium-voltage load. The energy balance procedure inside the converter was discussed and it was shown that the MMC can regulate all of the DC capacitor voltages at a predetermined level while the output currents are unbalanced and distorted. An appropriate predictive current controller which predicts in real time the module voltages, presents ac currents of the MMC based STATCOM that track their references with a small ripple. Considering the simulation studies, to compensate higher order harmonics, a smaller switching frequency for each switch is needed. This will result in smaller switching power losses and an improvement in energy efficiency, as well as a reduction in the heat dissipation, dimensions and weight of the converter. The main advantages of the MMC based STATCOM are its modularity and the ability to operation under unbalanced conditions without using a low frequency transformer. The intrinsic modularity characteristic of this topology increases its reliability and makes it suitable for high-power applications such as electrified railway power supply systems.

\section{REFERENCES}

[1] C. K. Lee, J.S K. Leung, S.Y R. Hui, H.S.H. Chung, "Circuit-level comparison of STATCOM technologies," IEEE Transactions on Power Electronics, Vol. 18, No. 4, pp. 1084-1092, Jul. 2003.

[2] Fang Z. Peng, John Wang, McKeever, Donald J. Adams, "A power line conditioner using cascade multilevel inverters for distribution systems," IEEE Transactions on Industrial Application, Vol. 34, No. 6, pp.12931298, Nov./Dec. 1998.

[3] Y. M. Park, H. S. Ryu, H. W. Lee, M. G. Jung, S. H. Lee, "Design of a cascaded H-Bridge multilevel inverter based on power electronics building blocks and control for high performance," Journal of Power Electronics, Vol. 10, No. 3, pp.262-269, Mar. 2010.

[4] H. I. Eini, S. Farhangi, J. L. Schanen, M. K. Fard, "A fault-tolerant control strategy for cascaded h-bridge multilevel rectifiers," Journal of Power Electronics, Vol. 10, No. 1, pp.34-42, Jan. 2010

[5] G. K. Kasal, B. Singh, "H-bridge VSC with a t-connected transformer for a 3-phase 4-wire voltage and frequency controller of an isolated asynchronous generator," Journal of Power Electronics, Vol. 9, No. 1, pp.43-50, Jan. 2009.

[6] H. Akagi, S. Inoue, T. Yoshii, " Control and performance of a transformerless cascade PWM STATCOM with star configuration," IEEE Transactions on Industry Applications, Vol. 43 , No. 4, pp. 1041 - 1049, Jul./Aug. 2007.

[7] Fang Z. Peng, Jin Wang, "A universal STATCOM with delta-connected cascade multilevel inverter," IEEE PESC'04, Jun. 2004.

[8] R.E. Betz, T. Summerst, T. Furney, "Using a cascaded h-bridge STATCOM for rebalancing unbalanced voltages," The 7th International Conference on Power Electronics, 2007.
[9] R. E. Betz, T. Summers, and T. Furney. "Symmetry compensation using a H-bridge multilevel STATCOM with zero sequence injection," IEEE Industry Applications Conference, Oct. 2006.

[10] R. Marquardt, A. Lesnicar, "New concept for high voltage - modular multilevel converter," Deutsch- Koreanisches Symposium, 2004.

[11] H. Mohammadi Pirouz, M. Tavakoli Bina, "New transformerless STATCOM topology for compensating unbalanced medium-voltage loads," EPE'09, Sep. 2009.

[12] Pee-Chin Tan, R.E. Morrison, D.G. Holmes, "Voltage form factor control and reactive power compensation in a $25-\mathrm{kV}$ electrified railway system using a shunt active filter based on voltage detection," IEEE Transactions on Industry Applications, Vol. 39, No. 2, pp. 575 - 581, Mar./Apr. 2003.

[13] R. Marquardt, "Stromrichters chaltungen mitverteilten energiespeichern," German Patent DE 10103 031, Jan. 24, 2001.

[14] M. Hiller, D. Krug, R. Sommer, S. Rohner, "A new highly modular medium voltage converter topology for industrial drive applications," 13th European Conference on Power Electronics and Applications, 2009.

[15] M. Hagiwara, H. Akagi, "Control and experiment of pulsewidthmodulated modular multilevel converters," IEEE Transactions on Power Electronics, Vol. 24, No. 7, pp 1737 - 1746, Jul. 2009.

[16] M. Tavakoli Bina, A.K.S Bhat, "Averaging technique for the modeling of STATCOM and active filters," IEEE Transactions on Power Electronics, Vol. 23, No. 2, pp. 723 - 734, Mar. 2008.

[17] Akagi, Watanabe, Aredes, "Instantaneous power theory and applications to power conditioning," The Institute of Electrical and Electronics Engineers Inc., 2007.

[18] R. Gupta, A. Ghosh, and A. Joshi, "Switching characterization of cascaded multilevel-inverter-controlled systems," IEEE Transactions on Industrial Electronics, ol. 55, no. 3, pp. 1047-1058, Mar. 2008.

[19] S. Rohner, S. Bernet, M. Hiller, R. Sommer, "Modulation, losses and semiconductor requirements of modular multilevel converters," IEEE Transactions on Industrial Electronics, IEEE Early Access, 2009.

[20] L. A. Moran, J. W. Dixon, and R. R. Wallace, "A three-phase active power filter operating with fixed switching frequency for reactive power and current harmonic compensation," IEEE Trans. Ind. Electron., Vol. 42, No. 4, pp. 402-408, Aug. 1995.

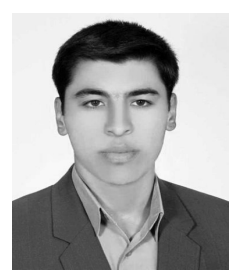

Hassan Mohammadi Pirouz (S'08) was born in Mashhad, Iran, on December 28, 1980. He received his M.S. from the K. N. Toosi University of Technology in September 2005. He is currently a Ph.D. student at the K. N. Toosi University. His research interests are in the areas of designing, modeling and control of high power electronics converters and their applications in FACTS controllers. Mr. Pirouz is a student member of IEEE and The Institute of Engineering and Technology

(IET).

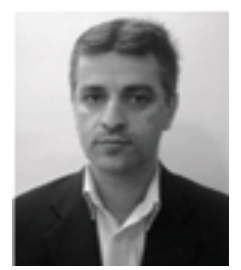

Mohammad Tavakoli Bina (S'98, M'01, SM'07) was born in Tehran, Iran, on July 14, 1962. He received his B.S. from the University of Teheran in 1992, and his Ph.D. in Power Electronics and Power System Interconnection from the University of Surrey in the UK in June 2001. He has been a certified engineer in the province of Tehran for ten years. He is presently holding an Associate Professor position at the K. N. Toosi University of Technology. His research interests include design and control of power electronic applications in power systems. 